\title{
Downregulation of occludin affects the proliferation, apoptosis and metastatic properties of human lung carcinoma
}

\author{
MEIFANG WANG $^{1,2}$, YUQUAN LIU ${ }^{2}$, XIN QIAN $^{2}$, NA WEI $^{2}$, YIJUN TANG $^{2}$ and JIONG YANG ${ }^{1}$ \\ ${ }^{1}$ Department of Respiratory and Critical Care Medicine, Zhongnan Hospital of Wuhan University, Wuhan, Hubei 430071; \\ ${ }^{2}$ Department of Respiratory Medicine, Taihe Hospital, Hubei University of Medicine, Shiyan, Hubei 442000, P.R. China
}

Received November 6, 2017; Accepted April 17, 2018

DOI: 10.3892/or.2018.6408

\begin{abstract}
Lung cancer is the most frequent and deadliest cancer in the world, especially in China. However, the molecular mechanisms involved in lung cancer remain unclear. Occludin (OCLN), one of the first identified tight junction proteins, has been revealed to be a necessary integral protein for tight junction structure and function. In the present study, we investigated the role of occludin in lung tumorigenesis. We found that occludin protein expression was highly increased in human lung cancer patient samples. Western blotting results also revealed that occludin expression was different in several lung cancer cell lines, with the highest level in SPC-A1 cells. Moreover, occludin knockdown inhibited lung cancer cell proliferation in vitro and in vivo. In addition, occludin knockdown promoted the apoptosis of lung cancer cell lines and reduced the invasion ability. Mechanistically, the activity of key growth pathway AKT/PI3K was compromised after occludin knockdown. Expression of apoptosis-related proteins, BAX, caspase-3, caspase- 9 and AIF, but not Bcl-2, were upregulated after silencing of occludin. Collectively, our findings for the first time identify the role of occludin as a tumor promoter and a pro-metastatic factor in lung cancer, demonstrating that occludin is a potential prognostic biomarker and therapeutic target in lung cancer.
\end{abstract}

\section{Introduction}

Lung cancer is the most common cancer worldwide (1). Moreover, it is the first leading cause of cancer-related deaths in China and is a major public health problem, due to limited therapeutic options in advanced stages (2). In addition, metastasis, which is formed by the spread of disseminated primary tumor cells to distant anatomic sites, leads to more than $90 \%$

Correspondence to: Dr Jiong Yang, Department of Respiratory and Critical Care Medicine, Zhongnan Hospital of Wuhan University, 169 Donghu Road, Wuhan, Hubei 430071, P.R. China

E-mail: yangjiongwh@126.com

Key words: lung cancer, occludin, proliferation, apoptosis, metastasis of the deaths of cancer patients $(3,4)$. Fundamentally, aberrant processing of genetic information, involving activation of oncogenes coupled with inactivation of tumor-suppressor genes, can lead to the carcinogenesis of lung cancer (5). In view of this, the discovery of novel molecular targets involved in lung cancer development and metastasis is critical to develop diagnostic markers and targeted therapies.

Since cancer cells must pass through a series of barriers in order to successfully metastasize to secondary foci, the tight junction (TJ) has become a crucial component in the process of cancer metastasis over the past decade $(6,7)$. One of the first identified TJ proteins is occludin (OCLN), which has been revealed to be a necessary integral protein for TJ structure and function (8). Occludin, widely expressed in tissues and cells that have TJs, is a membrane protein with four trans-membrane domains forming two extracellular loops and a long cytoplasmic tail $(9,10)$. Although occludin can bind directly to actin (11), cytoplasmic scaffolding protein zonaoccludens ( $\mathrm{ZO})-1$ can also serve as a link between occludin and the actin cytoskeleton (10). However, accumulating evidence has revealed that the claudin family, containing more than 20 members, is the core component of TJs (12). Apart from the basic function, TJs have also been demonstrated to be associated with signal transduction such as cancer related mitogen activated protein kinase (MAPK) and Akt signaling pathways (13), which further implies its important role in cancer development.

As anticipated, previous studies revealed that dysregulation of TJ function is vital to the development and invasiveness of many cancers $(14,15)$. Consistently, high expression of $\mathrm{TJ}$-associated proteins has been revealed to promote apoptosis and inhibit the metastatic potencies of these cancer cells (16). These data revealed that the TJ-related proteins may function in a context-dependent manner. However, the role of occludin in lung cancer development and metastasis remains to be elucidated. In the present study, we silenced occludin in lung cancer cells successfully, and observed suppressed cell proliferation and increased apoptosis, as well as compromised invasion ability. Our findings indicate that occludin is a likely candidate of oncogenes in lung cancer.

\section{Materials and methods}

Clinical tissue specimens. Fresh lung cancer tissue samples and corresponding adjacent normal tissues were obtained from 
patients with lung cancer who underwent surgical resections at Zhongnan Hospital of Wuhan University from March 2014 to December 2015. These samples were immediately frozen in liquid nitrogen and preserved at $-80^{\circ} \mathrm{C}$ freezer. All the experiments were carried out in accordance with the approved guidelines of Hubei University of Medicine.

Cell culture. Lung cancer cell lines including A549, NCL-H1650, SPC-A1, HCC827, NCI-H1299, and MSTO-211H were obtained from the Cell Bank of the Chinese Academy of Sciences (Shanghai, China). All cells were cultured in Dulbecco's modified Eagle's medium (DMEM) with 10\% fetal bovine serum (FBS; both from HyClone Laboratories; GE Healthcare Life Sciences, Logan, UT, USA). All cells were incubated at $37^{\circ} \mathrm{C}$ in $5 \% \mathrm{CO}_{2}$ humidified air.

siRNA transfection. For knockdown of occludin in lung cancer cell lines, siRNA was used. The sequences of the oligonucleotides were as follows: Control siRNA, CCUUUU AGGAGGUAGUGUAtt; occludin siRNA, GGUUCUGGU GUGAACUAAAtt. For transfection, cells were seeded in a 12 -well plate with a density of $5 \times 10^{5}$ cells/well. After $24 \mathrm{~h}$ and $70-80 \%$ confluence, the cells were transfected with respective occludin siRNAs (50 $\mathrm{nM})$ and control-siRNA (50 $\mathrm{nM})$ in serum-free medium using Lipofectamine $2000^{\mathrm{TM}}$ (Thermo Fisher Scientific, Inc., Waltham, MA, USA) according to manufacturer's instructions. After incubation for $6 \mathrm{~h}$ at $37^{\circ} \mathrm{C}$, the medium in each well was then replaced with DMEM, with $10 \%$ heat-inactivated FBS for the indicated time-points.

Real-time PCR analysis. Total RNA was extracted from lung cancer cell lines using TRIzol ${ }^{\circledR}$ Reagent (Invitrogen; Thermo Fisher Scientific, Inc.). Then RNase-free DNase was used to remove genome DNA. Reverse transcription was performed using AMV reverse transcriptase (Promega Corp., Madison, WI, USA) as well as a combination of random oligo(dT) primers according to the manufacturer's instructions. For the detection of the indicated gene expression in RNA levels, real-time PCR with SYBR-Green detection was performed. The primer pair used for amplification of the human occludin gene was as follows: Forward primer, 5-ACAAGCGGTTTT ATCCAGAGTC-3 and reverse primer, 5-GTCATCCACAGG CGAAGTTAAT-3. As an internal standard, human actin was amplified using the following primers: Forward primer, 5-GTGGACATCCGCAAAGAC-3 and reverse primer, 5-AAAGGGTGTAACGCAACTA-3. The real-time PCR reaction systemwas as follows: $10 \mu \mathrm{l}$ of $2 \mathrm{X}$ SYBR Premix Ex Taq, $0.8 \mu \mathrm{l}$ of forward and reverse primers $(2.5 \mu \mathrm{M}), 5 \mu \mathrm{l}$ of cDNA and $4.2 \mu \mathrm{l}$ of $\mathrm{ddH}_{2} \mathrm{O}$. The real-time PCR cycling conditions were as follows: Initial denaturation at $95^{\circ} \mathrm{C}$ for $1 \mathrm{~min}$; denaturation at $95^{\circ} \mathrm{C}$ for $5 \mathrm{sec}$; annealing extension at $60^{\circ} \mathrm{C}$ for $20 \mathrm{sec}$ (a total of 40 cycles). All of the reactions were performed using the Bio-Rad Connect Real-Time PCR platform (Bio-Rad Laboratories, Hercules, CA, USA). Absorbance valueswere read at the extension stage and all data were analyzed using the $2^{-\Delta \Delta \mathrm{Ct}}$ method.

Western blot analysis. Cells were lysed for total protein extraction in $2 \mathrm{X}$ SDS sample buffer $[100 \mathrm{mM}$ Tris- $\mathrm{HCl}$ (pH 6.8), 10 mM EDTA, 4\% SDS, 10\% glycine]. A total of
$30 \mu \mathrm{g}$ denatured proteins, were run on $12 \%$ polyacrylamide gels containing SDS and electroblotted onto nitrocellulose membranes. Membranes were then blocked with 5\% non-fat dry milk and were immunoblotted with antibodies overnight. After extensive washing, the membranes were reacted with peroxidase-labeled corresponding secondary antibodies in PBST and again washed; finally, the immunoreactions were visualized by using an enhanced chemiluminescence (ECL) system (Thermo Fisher Scientific, Inc., Waltham, MA, USA). Primary antibodies to occludin (cat. no. ab167161), caspase-3 (cat. no. ab44976), caspase-9 (cat. no. ab2013) and AIF (cat. no. ab1998) were purchased from Abcam (Cambridge, UK) with 1:1,000 dilutions. Primary antibodies to BAX (cat. no. sc-493) and Bcl-2 (cat. no. sc-492) were purchased from Santa Cruz Biotechnology, Inc. (Dallas, TX, USA) with 1:200 dilutions. Primary antibodies to cytochrome $c$ (cyt $c$; cat. no. 11940), p-AKT (cat. no. 4060), AKT (cat. no. 2920), p-PI3K (cat. no. 4228), PI3K (cat. no. 4257) and GAPDH (cat. no. 5174) were purchased from Cell Signaling Technology, Inc. (Danvers, MA, USA) with 1:1,000 dilutions. Secondary antibodies: Anti-rabbit HRP (cat. no. sc-2370) and anti-mouse HRP (cat. no. sc-2371) were obtained from Santa Cruz Biotechnology, Inc. with 1:5,000 dilutions.

$C C K-8$ viability assay. The growth of cells was assessed by the CCK-8 assay. An equal number of 2,000 cells were seeded in 96-well plate and cultured for various durations after siRNA transfection. At indicated time-points $(24,48,72,96$ and $120 \mathrm{~h}$ ), $10 \mu \mathrm{l}$ of CCK-8 (Dojindo Molecular Technologies, Inc., Kumamoto, Japan) was added to the cell medium and incubated for another $1 \mathrm{~h}$ at $37^{\circ} \mathrm{C}$. The absorbance was assessed quantitatively at $450 \mathrm{~nm}$ on an ELISA platereader (Model 680; Bio-Rad Laboratories).

Flow cytometric apoptosis analysis. SPC-A1 cells $(250,000$ cells/dish) were seeded in a $10-\mathrm{cm}$ dish after siRNA transfection. Forty-eight hours later, the cells were also harvested, washed in PBS followed by staining with Annexin V and 7-AAD (Dojindo Molecular Technologies, Inc.) for $30 \mathrm{~min}$ when cell density reached $80 \%$. The samples were then analyzed using a flow cytometer. The proliferation indices were analyzed using ModFit software (Verity Software House, Inc., Topsham, ME, USA) and were evaluated according to the formula $(\mathrm{S}+\mathrm{G} 2) /(\mathrm{G} 1+\mathrm{S}+\mathrm{G} 2)$.

Flow cytometric cell cycle analysis. SPC-A1 cells $\left(3 \times 10^{6}\right)$ were seeded in $10-\mathrm{cm}$ dishes and transfected with siRNA for $48 \mathrm{~h}$. Cells were harvested and washed twice with cold PBS and then fixed with ice-cold $70 \%$ ethanol for $1 \mathrm{~h}$ at $4^{\circ} \mathrm{C}$. After centrifugation at $1,000 \mathrm{x} \mathrm{g}$ for $5 \mathrm{~min}$, the cells were washed twice with $\mathrm{PBS}$ and resuspended with $0.5 \mathrm{ml}$ of PBS containing PI $(50 \mu \mathrm{g} / \mathrm{ml})$ and RNase $(100 \mu \mathrm{g} / \mathrm{ml})$. The cells were then stained at room temperature in the dark for $15 \mathrm{~min}$, and the cell cycle distribution was assessed by flow cytometry.

Transwell assays. To assess the invasiveness of lung cancer cells, we used Trans well chambers (Corning Inc., Corning, NY, USA) coated with Matrigel (BD Biosciences, Franklin Lakes, NJ, USA). Fully trypsinized transfected cells 
A
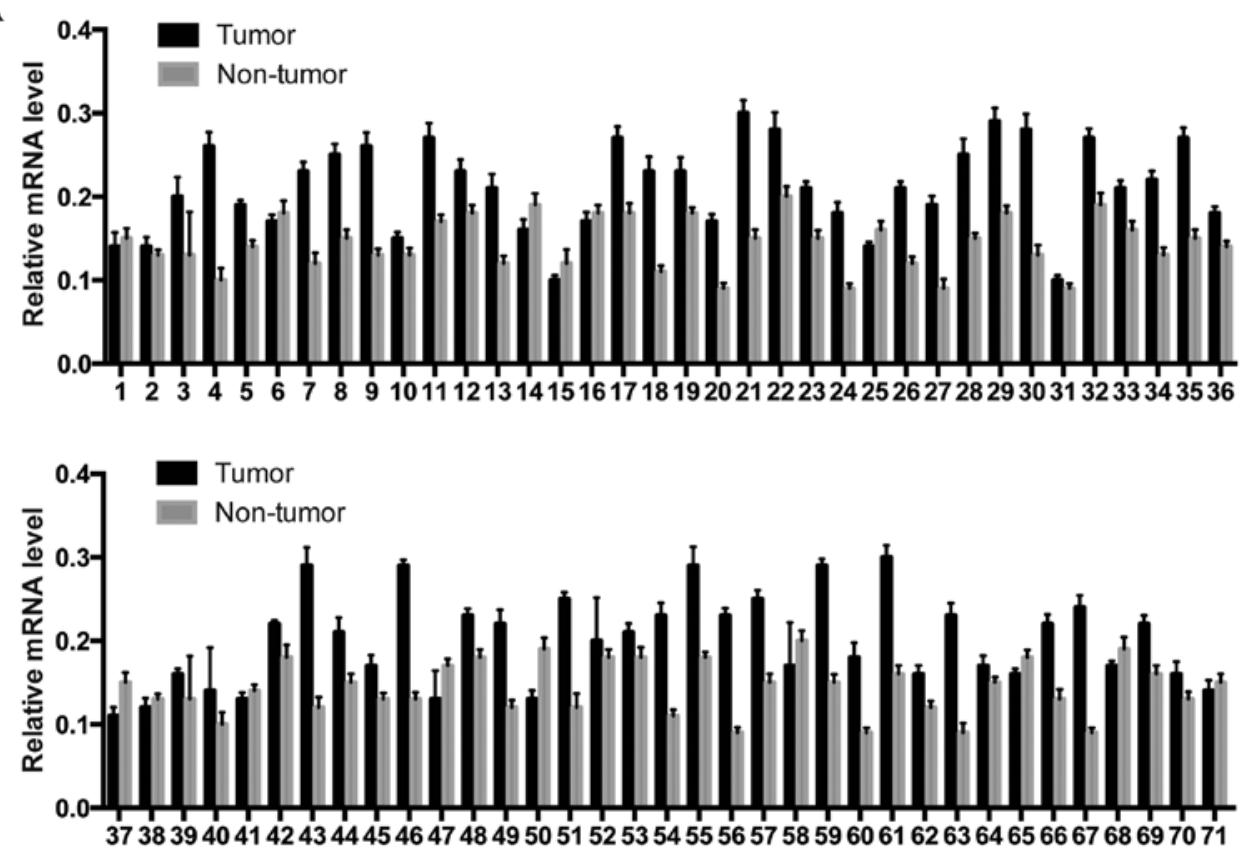

B
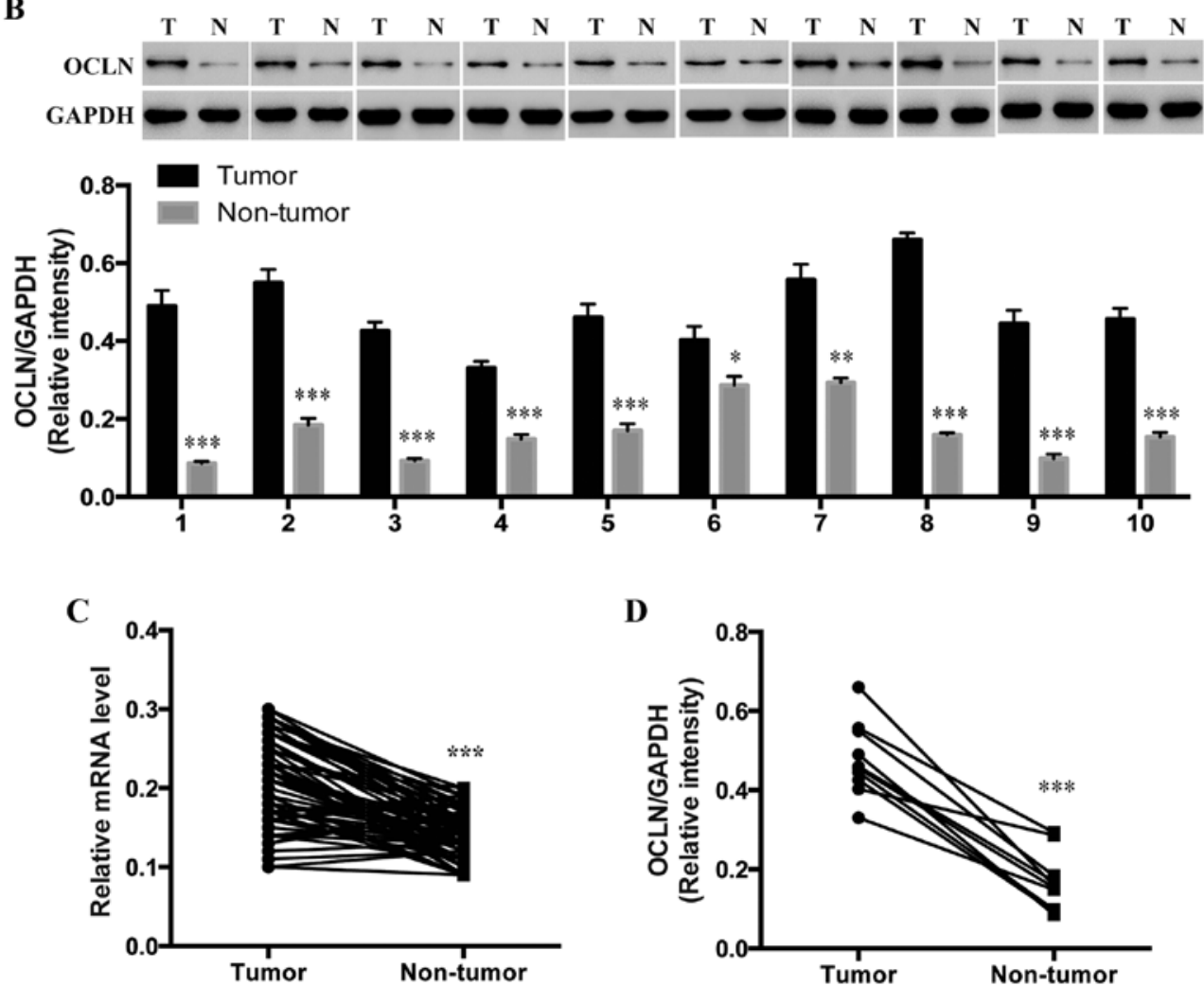

Figure 1. Occludin is overexpressed in lung cancer tumor tissues. (A) qRT-PCR was performed to assess the mRNA level of occludin in lung cancer tumor tissues and paired adjacent normal tissues. GAPDH was used as a control. (B) Western blottingwas used to assess the protein level of occludin in lung cancer tumor tissues and paired adjacent normal tissues. GAPDH was used as a control. (C) Quantification of the expression level of occludin in the two groups and normalized to GAPDH. (D) Quantification of the expression level of occludin in the two groups (by the grayscale value in A) and normalized to GAPDH. Error bars denote standard deviations. $\mathrm{n}=10,{ }^{*} \mathrm{P}<0.05,{ }^{* *} \mathrm{P}<0.01,{ }^{* * * *} \mathrm{P}<0.001$. OCLN, occludin.

( $1 \times 10^{5}$ cells in $100 \mu 1$ of serum-free DMEM) were plated into the upper chamber. DMEM $(500 \mu \mathrm{l})$ supplemented with $20 \%$ FBS was added to the lower chamber. After incubation in a humidified atmosphere containing $5 \% \mathrm{CO}_{2}$ at $37^{\circ} \mathrm{C}$ for $72 \mathrm{~h}$, lung cancer cells that had invaded the lower chamber were fixed with methanol and stained with crystal violet. We used an inverted microscope to capture images of the invading cells manually. A Transwell assay was also performed to assess the cell migration in the same manner as the Transwell invasion assay with the exception of Matrigel coating. 
A

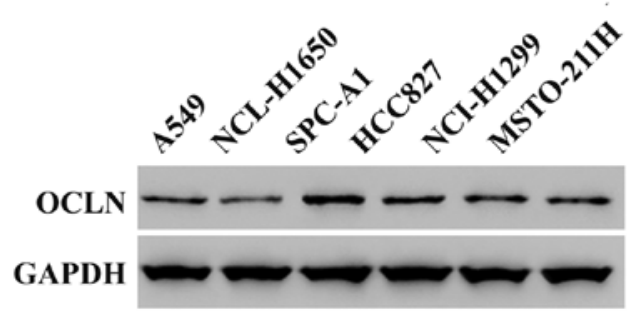

B

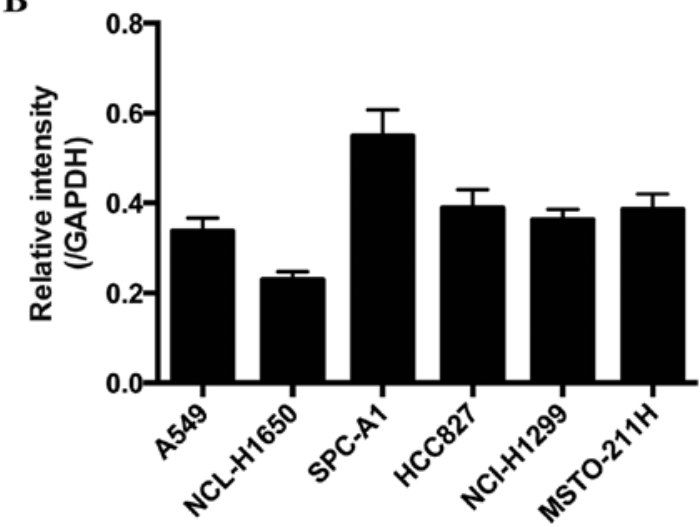

E

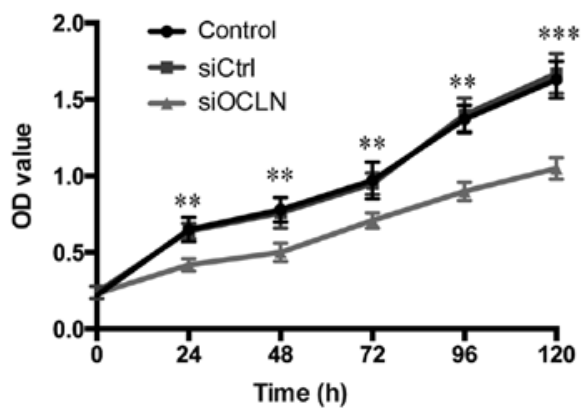

C

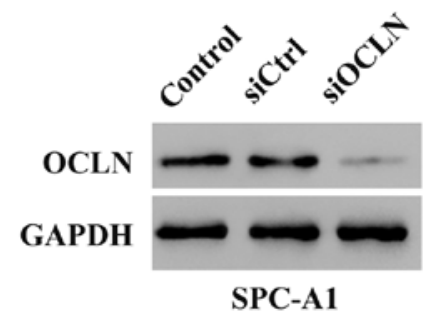

D

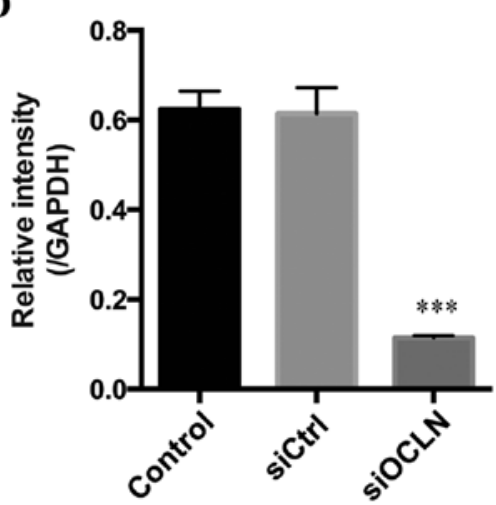

Figure 2. Occludin knockdown inhibits cell proliferation in vitro. (A) Western blotting was used to assess the protein level of occludin invarious lung cancer cell lines. GAPDH was used as a control. (B) Quantification of the expression value of occludin in cell lines (by the grayscale value in A) and normalized to GAPDH. (C) Western blotting was used to assess the protein level of occludin in lung cancer cellline SPC-A1. GAPDH was used as a control. (D) Quantification of the expression level of occludin in SPC-A1 (by the grayscale value in C) and normalized to GAPDH. (E) CCK-8 growth assay of the control, siCtrl, and siOCLN of SPC-A1 cells. ${ }^{* *} \mathrm{P}<0.01,{ }^{* * *} \mathrm{P}<0.001$. OCLN, occludin.

Mouse xenograft model. A mouse xenograft model was established as previously described (17). Briefly, Balb/c (nu/nu) (4-5 weeks old) nude mice were randomly divided into two groups and subcutaneously injected in the flank regions with $1.0 \times 10^{7}$ cells in $0.1 \mathrm{ml}$ of PBS. The tumor size was assessed every 4 days with calipers and the tumor volume was calculated with the formula: (length $\mathrm{x}$ width ${ }^{2}$ )/2. Forty days following implantation, the mice were euthanized by asphyxiation in $\mathrm{CO}_{2}$ chamber and tumors were excised immediately and images were captured. All procedures were conducted in accordance to the Animal Care and Use Committee Guidelines of Hubei University of Medicine.

Statistical analysis. All data were presented as the mean \pm standard deviation (SD) and normalized relative to the negative control. Statistical analysis was performed using Prism 6 software. Differences among different groups were analyzed by one-way ANOVA. A P-value $<0.05$ was considered to indicatea statistically significant difference.

\section{Results}

Occludin is overexpressed in lung cancer tumor tissues. To determine whether occludin is involved in lung cancer development, we collected 71 pairs of lung cancer tumor tissues and their paired adjacent normal tissues and performed qRT-PCR to assess the relative expression levels of occludin. Notably, the relative expression level of occludin in the lung cancer tissues was significantly higher than that in the corresponding adjacent non-tumor tissues (Fig. 1A and C). Furthermore, in a small set of human lung cancer tumor and normal tissues from the same patients, all of the 10 tumor tissues exhibited increasedoccludin protein levels (Fig. 1B and D), which indicates that occludin may participate in the pathogenesis of lung cancer. 
A

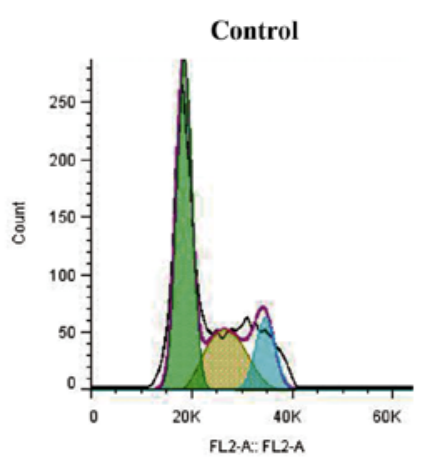

B

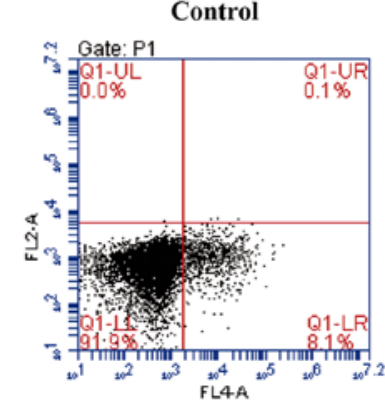

C

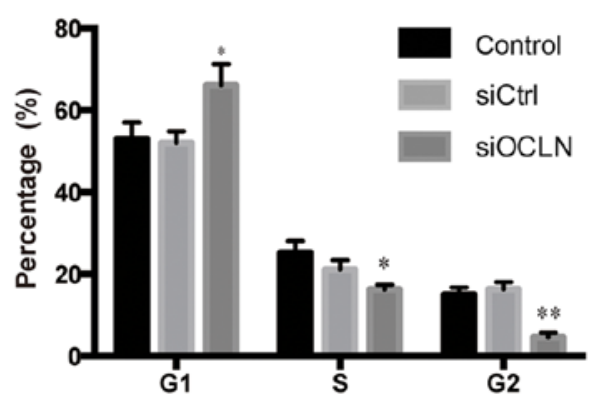

siCtrl

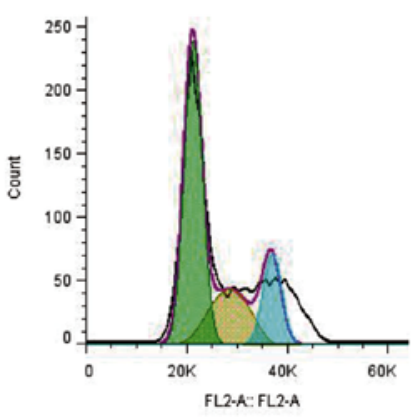

siCtrl

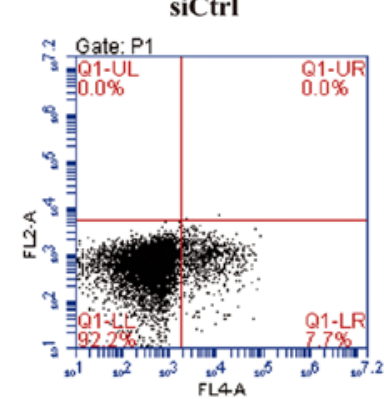

D

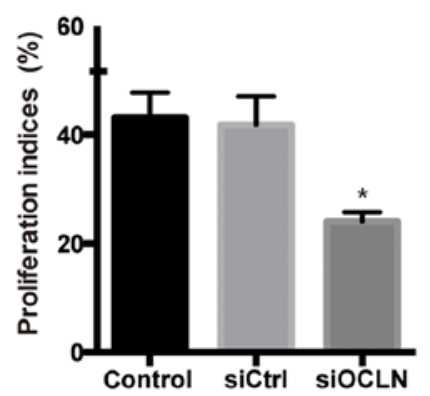

SiOCLN

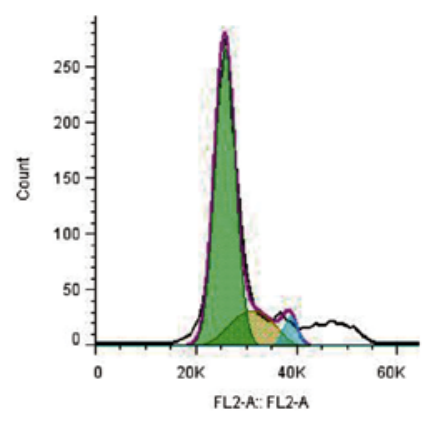

SiOCLN

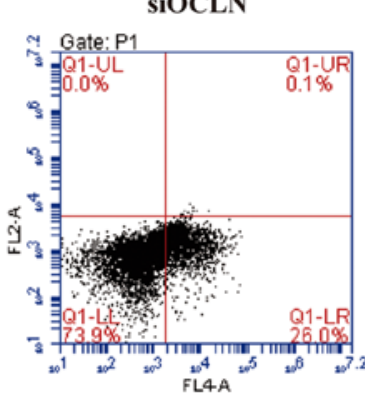

E

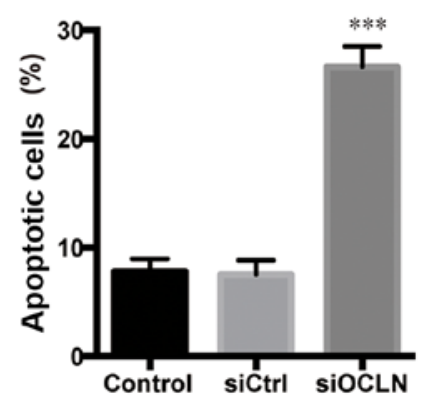

Figure 3. Occludin silencing induces cell cycle arrest and cell apoptosis. (A) Flow cytometry was used to assess the cell cycle distribution of SPC-A1 cells stained with PI. Representative resultsare shown. (B) Flow cytometry was used to assess the percentage of apoptotic SPC-A1 cells after tranfection with scramble or occludin siRNA. Representative scatter plots are shown. (C) Quantification of the percentage of G1, S, G2 cells assayed by flow cytometry. (D) Calculation of the proliferation indices according to flow cytometry. (E) Quantification of the percentage of apoptotic SPC-A1 cells. ${ }^{*} \mathrm{P}<0.05$, ${ }^{* *} \mathrm{P}<0.01$, **** $\mathrm{P}<0.001$. OCLN, occludin

Occludin knockdown inhibits cell proliferation. To analyze the expression pattern of occludin in lung cancer cells, six lung cancer cell lines, including A549, NCL-H1650, SPC-A1, HCC827, NCI-H1299 and MSTO-211H were examined. We determined that occludin was highly expressed in SPC-A1, moderately expressed in A549, HCC827, NCI-H1299 and MSTO-211H while relatively lowly expressed in NCL-H1650 cells (Fig. 2A and B). Therefore, we selected the SPC-A1 cell line to perform the following experiments. To investigate the functional role of occludin in lung carcinogenesis, siRNA-mediated loss-of-function experiments in vitro were performed. The occludin expression levels of SPC-A1 cells transfected with occludin siRNA were significantly lower than that of SPC-A1 cells transfected with the scrambled sequence (Fig. 2C and D), indicating high knockdown efficiency.

Next, we evaluated the effect of occludin on lung cancer cell proliferation in vitro by CCK-8 assay. Notably, we found that occludin knockdown resulted in significantly decreased cell proliferation in SPC-A1 cells at every time-point, indicating that occludin has a role in promoting the proliferation of SPC-A1 cells (Fig. 2E). These results demonstrated that overexpression of occludin promoted the proliferation of lung cancer cells.

Occludin silencing induces cell cycle arrest and cell apoptosis. We next investigated whether occludin promotes the proliferation of SPC-A1 cells by influencing cell cycle distribution in vitro. The cell cycle analysis revealed that, compared with transfection with the scrambled siRNA, transfection with occludin siRNA resulted in a significant reduction of the SPC-A1 cells in the S and G2 phases and an increase in the G1 phase (Fig. 3A and C). Furthermore, we found that knockdown occludin significantly decreased the proliferation indices (Fig. 3D). Collectively, we demonstrated that the increase of occludin contributed to the proliferation of lung cancer cells by promoting cell cycle progression. 
A

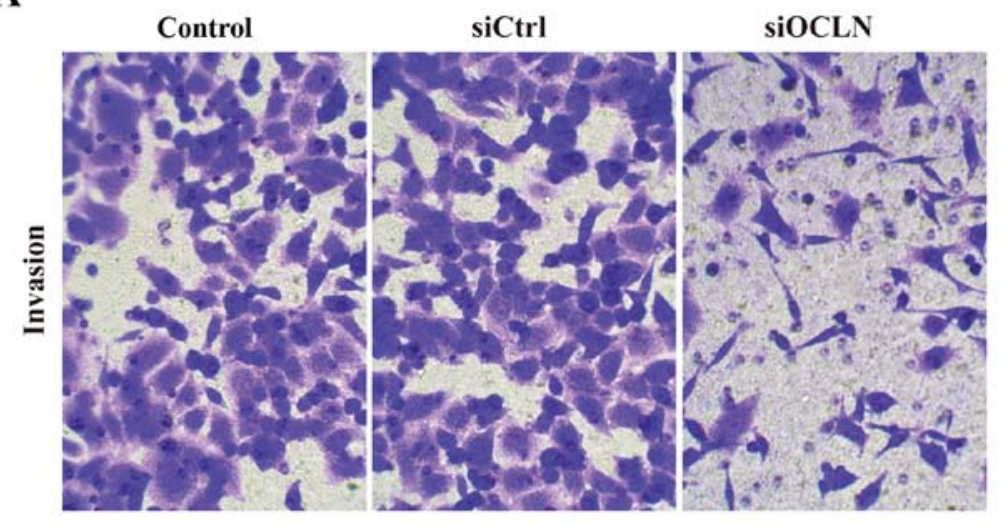

C

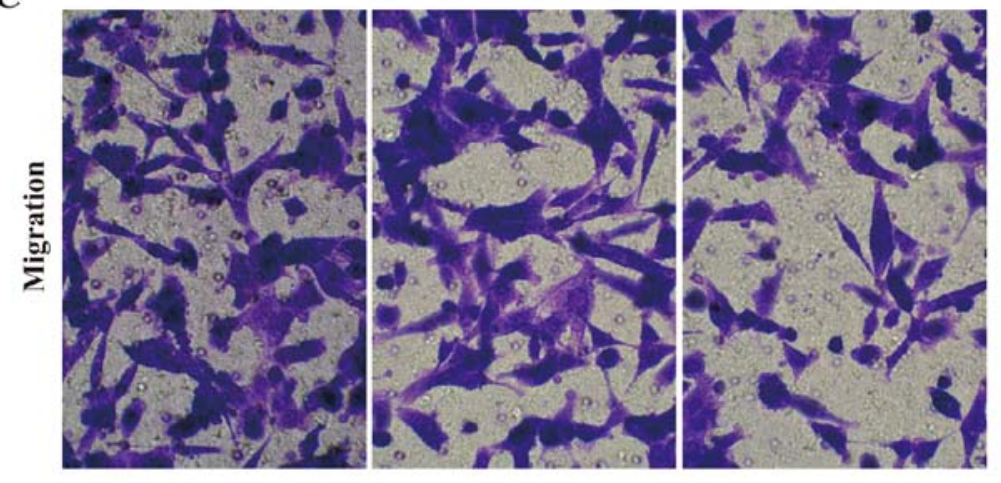

B

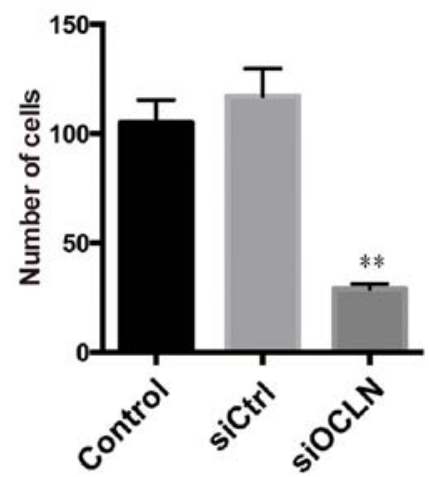

D

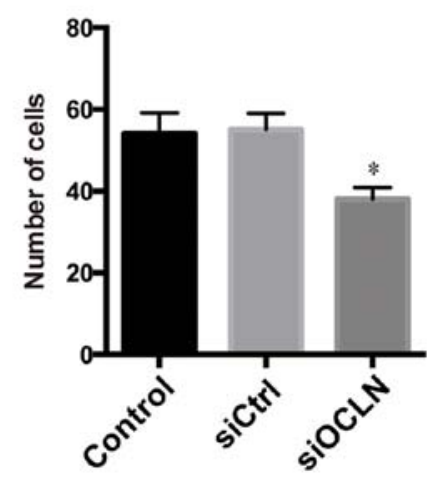

Figure 4. Occludin silencing inhibits cell invasion and migration. (A and B) A Transwell assay was conducted to assess the invasion of SPC-A1 cells transfected with scramble or occludin siRNA. Representative images and quantification of the cells are shown. (C and D) A Transwell assay was conducted to assess the migration of SPC-A1 cells transfected with scramble or occludin siRNA. Representative images and quantification of the cells are shown. ${ }^{*} \mathrm{P}<0.05,{ }^{* *} \mathrm{P}<0.01$. OCLN, occludin.

A

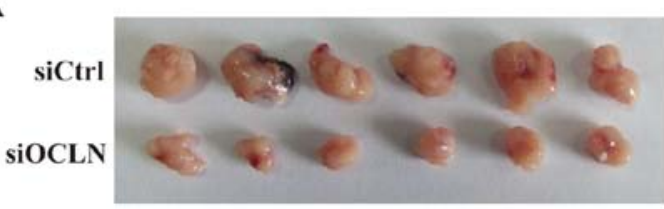

B

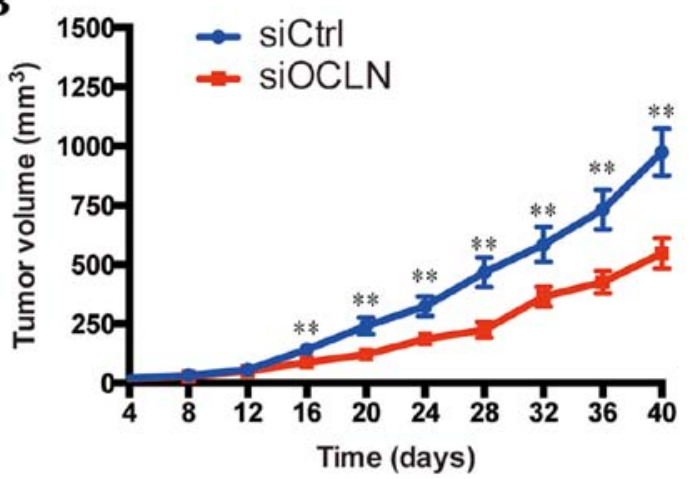

Figure 5. Occludin silencing inhibits lung cancer cell proliferation in a xenograft tumor model in vivo. (A) Forty days after subcutaneous injections of SPC-A1 cells transfected with scramble or occludin siRNA into the dorsal flanks of female nude mice, tumors were removed at necropsy and photographed. (B) Growth of xenograft-induced tumors was assessed externally at indicated time-points; tumor volumes were calculated and the mean volumes $\pm \mathrm{SD}$ are shown ( $\mathrm{n}=6$ ). ${ }^{* * *} \mathrm{P}<0.01$. OCLN, occludin.

We further examined the effect of occludin on apoptosis using flow cytometry. As anticipated, the early apoptotic cells (Annexin $\mathrm{V}^{+} / 7 \mathrm{AAD}^{-}$) were significantly increased after occludin knockdown in SPC-A1 cells (Fig. 3B and D), indicating that plays an anti-apoptotic role. In summary, we hypothesized that occludin supports lung cancer growth by exerting anti-apoptotic effects and promoting cell cycle progression in vitro.
Occludin silencing inhibits cell invasion and migration. Considering that $\mathrm{TJ}$ proteins are significantly correlated with the metastasis of various cancers, we further studied the role of occludin in the regulation ofinvasion ability by Transwell assays. Knockdown of occludin in SPC-A1 cells markedly attenuated their invasive ability (Fig. 4A and B), suggesting that occludin has a critical function in improving the invasion of lung cancer cells. Next, we explored the effect of occludin on 
A

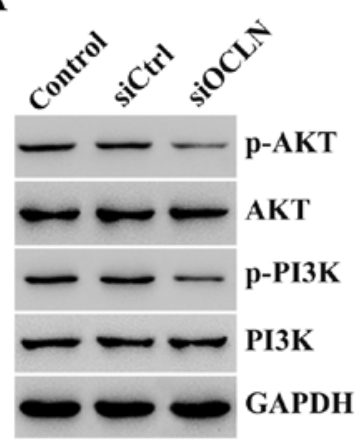

C

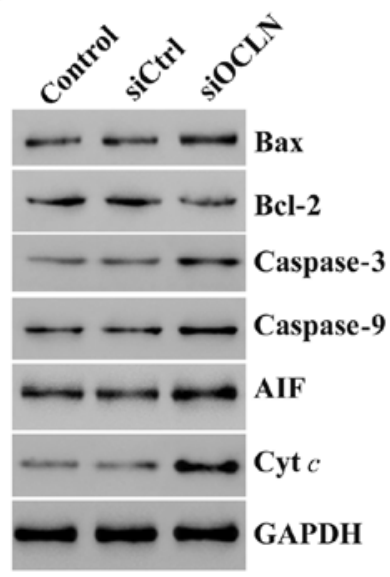

B
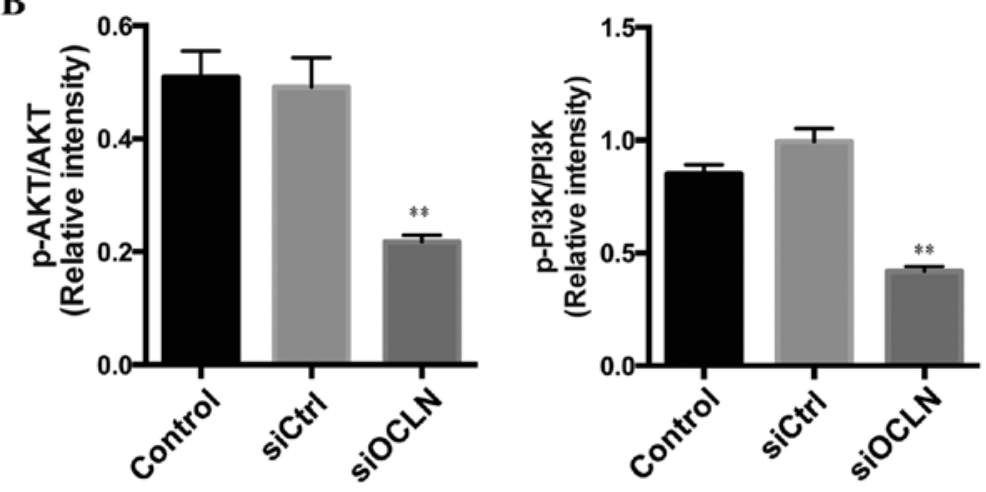

D

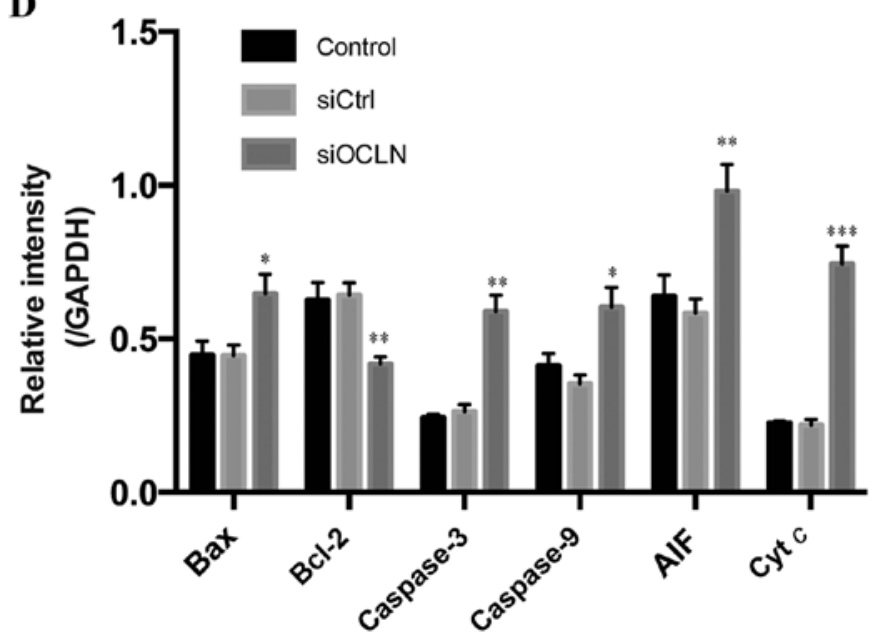

Figure 6. Occludin knockdown regulates apoptosis-related proteins and AKT/PI3K signaling. (A) Western blotting was used to assess the protein level of p-AKT, AKT, p-PI3K, PI3K in the control, siCtrl, and siOCLN of SPC-A1 cells. GAPDH was used as a control. (B) Quantification of the p-AKT and p-PI3K level in cell lines (by the grayscale value in A) and normalized to AKT and PI3K correspondingly. (C) Western blotting was used to assess the protein level of BAX, caspase-3, caspase-9, AIF, and Bcl-2 in the control, siCtrl, and siOCLN of SPC-A1 cells. GAPDH was used as a control. (D) Quantification of BAX, caspase-3, caspase-9, AIF, and Bcl-2 level in SPC-A1 (by the grayscale value in C) and normalized to GAPDH. ${ }^{*} \mathrm{P}<0.05,{ }^{* *} \mathrm{P}<0.01,{ }^{* * *} \mathrm{P}<0.001 . \mathrm{OCLN}$, occludin.

cell migration and found that occludin silencing significantly decreased the migration capacity as well (Fig. 4C and D). Therefore, we determined that occludin was a pro-metastatic factor in lung cancer cells.

Occludin silencing inhibits lung cancer cell proliferation in a xenograft tumor model in vivo. We further confirmed the aforementioned resultsin a xenograft tumor model in vivo. siCtrl and siOCLN SPC-A1 cells were injected subcutaneously into two groups of nude mice randomly. As anticipated, the tumor growth curve revealed that tumors derived from the siOCLN group grew more slowly than those from the siCtrl group (Fig. 5A). The tumor volume of the siCtrl group was $974 \pm 98.6 \mathrm{~mm}^{3}$, whereas the tumor volume of siOCLN group was $547 \pm 64.3 \mathrm{~mm}^{3} 40$ days after implantation (Fig. 5B). These results demonstrated that overexpression of occludin promoted the proliferation of lung cancer cells in vivo.

Occludin knockdown regulates apoptosis-related proteins and AKT/PI3K signaling. To discover the mechanisms of occludin in lung cancer growth, we investigated the proliferation and apoptosis-related signaling pathways. We found that the AKT/PI3K pathway activation, which is essential for most cell growth, was compromised after occludin silencing (Fig. 6A and B). Accordingly, we detected the protein level of apoptosis-related molecules and found that the expression of BAX, caspase-3, caspase-9, AIF, and cyt $c$ were upregulated while anti-apoptotic Bcl-2 was decreased in SPC-A1 cells transfected with occludin siRNA (Fig. 6C and D). Thus, we surmised that occludin regulated lung cancer cell growth by affecting the AKT/PI3K pathway and apoptosis-associated factors.

\section{Discussion}

Tight junctions (TJs) the most apical intercellular structures in epithelial and endothelial cells are considered to play important roles in the formation of cell polarity and paracellular permeability $(18,19)$. Additionally, accumulating evidence has revealed that the disruption of TJ structure has been linked to tumor development, which may be causally involved in malignant phenotypes, such as local tumor growth, invasion, and metastasis at distant sites $(6,7,14,15)$. In the present study, we first deduced the critical role of occludin, a crucial component of TJs, in lung cancer. Occludin, which was overexpressed in 
lung cancer tumor tissues, regulated the growth and metastasis of lung cancer cells in vitro and in vivo by controlling important proliferation pathways and a common set of cell apoptosis-related genes. Collectively, our study revealed the promotive role of occludin in lung cancer.

The two most fundamental traits of cancer cells involve their ability to sustain uncontrolled proliferation and resist cell death (5). An important finding of this study was the identification of occludin as a tumor promoter by altering the proliferation-associated pathways as well as the expression of apoptosis-related genes. The AKT/PI3K pathway, which is stimulated by insulin $(20,21)$, participates in cell proliferation among various cancer types. In our study, we revealed that occludin may promote lung cancer cell proliferation by modulating this key pathway. However, whether lung cancer development is associated with insulin levels remains unclear. Apoptosis is an evolutionarily conserved process in the regulation of tissue homeostasis during normal development. However, several studies have recognized that inadequate apoptosis leads to the malignant phenotype, since it is widely believed that impaired susceptibility to respond to numerous apoptotic signals is associated with oncogenic transformation (22-24). In the present stydy, we determined that occludin knockdown could increase apoptosis, which may be due to the altered apoptosis-related gene profiles. This is consistent with a previous study that revealed that occludin also regulates Bax, Bcl-2 and caspase-3 expression in liver cancer cell lines (25). Occludin-deficient mice also displayed pronounced numbers of apoptotic primary hepatocytes and immortalized cells stimulated by serum-free conditions compared with the wild-type cells (13), in line with our dataand the results in mammary gland cells (26). The modulation of the expression levels of these genes indicated that occludin may affect mitochondria-mediated apoptosis (27). However, the association between occludin and mitochondria function warrants further study in the future. In contrast, overexpression of occludin enhanced the sensitivity to $\mathrm{H}_{2} \mathrm{O}_{2}$-induced cell death in $\mathrm{HeLa}$ cells (28). Thus, the function of occludin in apoptosis is complex and may depend on the cell type. In summary, overexpression of occludin contributed to limitless proliferation as well as acquisition of apoptotic resistance of lung cancer cells and participated in the carcinogenic process accordingly.

It is well accepted that the metastatic process involves a complicated interplay between altered cell adhesion, survival, migration, and homing on distant target organs (29). Notably, loss of occludin also displayed suppressive and progressive cancer phenotypes, such as invasive properties, which resulted in an important contribution to metastatic inhibition. Therefore, our data revealed that forced expression of occludin in tumors favors various steps affecting lung cancer development. We could not exclude the possibility that occludin may have some unidentified but more notable effects on tumor cells. Thus, considering the complexity of carcinogenesis, the in vivo function of occludin in lung cancer warrants further exploration in the future.

In summary, we revealed that high occludin expression confers malignant growth of lung cancer cells largely by promoting proliferation and blocking apoptosis in vitro and in vivo, and suggests thatit may be a potential biomarker for lung cancer progression. Furthermore, our results revealed that occludin is a likely candidate as a tumor-promoter gene in certain types of cancer and may pave the way to new therapeutic modalities. Occludin depletion even antagonistically would be a potential therapeutic strategy for lung cancer.

\section{Acknowledgements}

Not applicable.

\section{Funding}

The present study was supported by the Educational Commission of Hubei Province of China (D20152104).

\section{Availability of data and materials}

The datasets used during the present study are available from the corresponding author upon reasonable request.

\section{Authors' contributions}

MW and JY conceived and designed the study. MW, YL, XQ, NW and YT performed the experiments. MW and JY wrote the paper. All authors read and approved the manuscript and agree to be accountable for all aspects of the research in ensuring that the accuracy or integrity of any part of the work are appropriately investigated and resolved.

\section{Ethics approval and consent to participate}

All experimental protocols were approved bythe Ethics Committee of Zhongnan Hospital of Wuhan University.

\section{Consent for publication}

Not applicable.

\section{Competing interests}

The authors state that they have no competing interests.

\section{References}

1. May M: Statistics: Attacking an epidemic. Nature 509: S50-S51, 2014.

2. Chen W, Zheng R, Baade PD, Zhang S, Zeng H, Bray F, Jemal A, $\mathrm{Yu}$ XQ and He J: Cancer statistics in China, 2015. CA Cancer J Clin 66: 115-132, 2016.

3. Fidler IJ: The pathogenesis of cancer metastasis: The 'seed and soil' hypothesis revisited. Nat Rev Cancer 3: 453-458, 2003.

4. Sone S and Yano S: Molecular pathogenesis and its therapeutic modalities of lung cancer metastasis to bone. Cancer Metastasis Rev 26: 685-689, 2007.

5. Hanahan D and Weinberg RA: Hallmarks of cancer: The next generation. Cell 144: 646-674, 2011.

6. Tobioka H, Sawada N, Zhong Y and Mori M: Enhanced paracellular barrier function of rat mesothelial cells partially protects against cancer cell penetration. Br J Cancer 74: 439-445, 1996.

7. Haynes MD, Martin TA, Jenkins SA, Kynaston HG, Matthews PN and Jiang WG: Tight junctions and bladder cancer (Review). Int J Mol Med 16: 3-9, 2005.

8. Furuse M, Hirase T, Itoh M, Nagafuchi A, Yonemura S, Tsukita S and Tsukita S: Occludin: A novel integral membrane protein localizing at tight junctions. J Cell Biol 123: 1777-1788, 1993 
9. Jiang WG, Bryce RP, Horrobin DF and Mansel RE: Regulation of tight junction permeability and occludin expression by polyunsaturated fatty acids. Biochem Biophys Res Commun 244: 414-420, 1998

10. Tsukita S, Furuse M and Itoh M: Multifunctional strands in tight junctions. Nat Rev Mol Cell Biol 2: 285-293, 2001.

11. Wittchen ES, Haskins J and Stevenson BR: Protein interactions at the tight junction. Actin has multiple binding partners, and ZO-1 forms independent complexes with ZO-2 and ZO-3. J Biol Chem 274: 35179-35185, 1999.

12. Aijaz S, Balda MS and Matter K: Tight junctions: Molecular architecture and function. Int Rev Cytol 248: 261-298, 2006.

13. Murata M, Kojima T, Yamamoto T, Go M, Takano K, Osanai M, Chiba $\mathrm{H}$ and Sawada N: Down-regulation of survival signaling through MAPK and Akt in occludin-deficient mouse hepatocytes in vitro. Exp Cell Res 310: 140-151, 2005.

14. Michl P, Barth C, Buchholz M, Lerch MM, Rolke M, Holzmann KH, Menke A, Fensterer H, Giehl K, Löhr M, et al: Claudin-4 expression decreases invasiveness and metastatic potential of pancreatic cancer. Cancer Res 63: 6265-6271, 2003.

15. Wang Z, Mandell KJ, Parkos CA, Mrsny RJ and Nusrat A: The second loop of occludin is required for suppression of Raf1-induced tumor growth. Oncogene 24: 4412-4420, 2005

16. Hoevel T, Macek R, Swisshelm K and Kubbies M: Reexpression of the TJ protein CLDN1 induces apoptosis in breast tumor spheroids. Int J Cancer 108: 374-383, 2004.

17. Li ZM, Tian T, Lv F, Chang Y, Wang X, Zhang L, Li X, Li L, $\mathrm{Ma} \mathrm{W}, \mathrm{Wu} \mathrm{J}$ and Zhang M: Six1 promotes proliferation of pancreatic cancer cells via upregulation of cyclin D1 expression. PLoS One n 8: e59203, 2013.

18. Schneeberger EE and Lynch RD: Structure, function, and regulation of cellular tight junctions. Am J Physiol 262: L647-L661, 1992.
19. Gumbiner BM: Breaking through the tight junction barrier. J Cell Biol 123: 1631-1633, 1993.

20. Burgering BM and Coffer PJ: Protein kinase B (c-Akt) in phosphatidylinositol-3-OH kinase signal transduction. Nature 376: 599-602, 1995.

21. Franke TF, Yang SI, Chan TO, Datta K, Kazlauskas A, Morrison DK, Kaplan DR and Tsichlis PN: The protein kinase encoded by the Akt proto-oncogene is a target of the PDGF-activated phosphatidylinositol 3-kinase. Cell 81: 727-736, 1995.

22. Fisher DE: Apoptosis in cancer therapy: Crossing the threshold. Cell 78: 539-542, 1994.

23. Evan GI and Vousden KH: Proliferation, cell cycle and apoptosis in cancer. Nature 411: 342-348, 2001.

24. Gozani O, Boyce M, Yoo L, Karuman P and Yuan J: Life and death in paradise. Nat Cell Biol 4: E159-E162, 2002.

25. Gu JM, Lim SO, Park YM and Jung G: A novel splice variant of occludin deleted in exon 9 and its role in cell apoptosis and invasion. FEBS J 275: 3145-3156, 2008.

26. Beeman NE, Baumgartner HK, Webb PG, Schaack JB and Neville MC: Disruption of occludin function in polarized epithelial cells activates the extrinsic pathway of apoptosis leading to cell extrusion without loss of transepithelial resistance. BMC Cell Biol 10: 85, 2009.

27. Wang X: The expanding role of mitochondria in apoptosis. Genes Dev 15: 2922-2933, 2001

28. Osanai M, Murata M, Nishikiori N, Chiba H, Kojima T and Sawada N: Epigenetic silencing of occludin promotes tumorigenic and metastatic properties of cancer cells via modulations of unique sets of apoptosis-associated genes. Cancer Res 66: 9125-9133, 2006

29. Bogenrieder T and Herlyn M: Axis of evil: Molecular mechanisms of cancer metastasis. Oncogene 22: 6524-6536, 2003. 